\title{
Cuidar de um familiar idoso dependente no domićlio: reflexões para os profissionais da saúde
}

\section{Proiding carefor adependert ddely redtivethone refletionsfor hedthprofessiands}

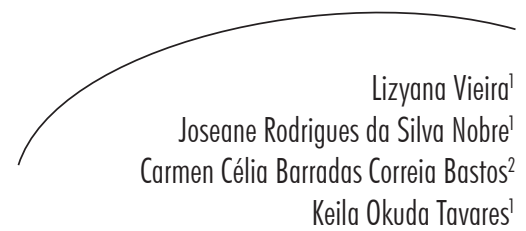

\section{Resumo}

Ao conduzir um estudo qualitativo de caráter fenomenológico, para compreender o que é cuidar de um idoso dependente por meio do olhar de seus familiares, foi possível observar vários aspectos dessa vivência. Dentre eles, chamou a atenção o fato de que esses cuidadores enfrentam sobrecarga física e mental. Objetivo: apresentar os resultados relacionados à sobrecarga física e mental relatadas pelos cuidadores familiares de idosos dependentes, com o intuito de fornecer informações que possam contribuir de forma positiva para a complementação do trabalho das equipes de saúde voltado para os idosos dependentes, levando em consideração que ele também deve ser voltado para a assistência dos cuidadores familiares. Método: estudo descritivo-exploratório, qualitativo, de caráter fenomenológico. Foram realizadas entrevistas com cuidadores familiares de idosos dependentes. A coleta de dados foi realizada por meio de uma entrevista semiestruturada, baseada em uma pergunta orientadora. As entrevistas foram gravadas e transcritas para serem analisadas. Resultados: o cuidador familiar de idosos dependentes é uma pessoa sobrecarregada do ponto de vista físico e mental, pelo acúmulo de funções e tipos de tarefas que assume. O estresse crônico relacionado a essa experiência pode alterar sua saúde. Conclusão: o fato de o cuidador familiar pertencer ao gênero feminino, possuir idade avançada e se responsabilizar sozinho por todos os cuidados e também o nível de capacidade funcional do idoso, são fatores que influenciam essa sobrecarga.

\section{Abstract}

By conducting a qualitative study of phenomenological approach, in order to understand what is caring for the dependent elderly in their relatives' viewpoint, it was possible to observe several aspects of this experience. Among them, we highlight that these caregivers are subjected to physical and mental overload. Objective: to present the results related to this overload, in order to provide information that contributes positively to

Palavras-chave: Cuidadores. Idoso Fragilizado. Família. Pessoal de saúde.
Key words: Caregivers. Frail Elderly. Family. Health personnel.

\footnotetext{
Departamento de Fisioterapia. Universidade Estadual do Oeste do Paraná. Cascavel, PR, Brasil.

Departamento de Pedagogia. Universidade Estadual do Oeste do Paraná. Cascavel, PR, Brasil.
} 
complement the work of health staff towards dependent elderly people, considering that it must care for the family caregivers. Method: this is a descriptive, exploratory, qualitative study of phenomenological approach. Interviews were conducted with family caregivers of dependent elderly people. Data was collected through semistructured interviews based on a guiding question. The interviews were taped and transcribed to further analysis. Results: the family caregiver of dependent elderly people is an overloaded person, both physically and mentally, due to the accumulation of functions and tasks that takes. The chronic stress related to this experience can change their health. Conclusion: the fact that the family caregivers are women, with advanced age, and have to be responsible alone for all the cares, and also the level of functional capacity of the elderly, are factors that influence this overload.

\section{INTRODUÇÃO}

A Organização Mundial da Saúde (OMS) considera idoso o indivíduo com idade igual ou superior a 60 anos. ${ }^{1} \mathrm{O}$ processo do envelhecimento acarreta alterações fisiológicas e, consequentemente, modificações na saúde, na funcionalidade física, no funcionamento intelectual, no exercício de papéis sociais e nas relações interpessoais dos indivíduos idosos. ${ }^{2}$

É comum que o ser humano tenha pelo menos uma patologia crônico-degenerativa nessa fase da vida, o que por sua vez pode provocar algum tipo de incapacidade e/ou dependência., ${ }^{3,4}$ Grande parte das doenças e limitações que os idosos apresentam ocasionam complicações e sequelas que comprometem sua capacidade funcional, a ponto de impedir o autocuidado, sobrecarregando a família e o sistema de saúde. ${ }^{5}$

No contexto familiar, a função de cuidador tende a ser assumida por uma única pessoa, denominada "cuidador principal". Esta assume e se responsabiliza pelo cuidado e representa o elo entre o idoso, o restante da família e a equipe de saúde. A designação do cuidador é informal, embora pareça que o processo atenda certas regras fundamentadas em fatores, como: parentesco (em sua maioria, os cônjuges), gênero (predominantemente a mulher), proximidade física (quem convive com o idoso) e proximidade afetiva (estabelecida pela relação conjugal e pela relação entre pais e filhos). ${ }^{6}$
O cuidado de um familiar doente $\mathrm{e}$ dependente no domicílio acontece no espaço onde parte considerável da vida é vivida, no qual o conhecimento e a memória de fatos e de relações íntimas são importantes, tanto para o cuidador, como para o idoso. Nesse ambiente, os cuidados têm suas particularidades. ${ }^{7}$ A preocupação sobre o cuidador familiar, que na maior parte dos casos nem sempre se encontra adequadamente preparado para essa prática, vem crescendo com o aumento do número de idosos que vivenciam um processo de doença crônica e incapacitante. ${ }^{8}$

Compreender a vivência de cuidar de um idoso dependente é importante para poder auxiliar de forma mais completa os familiares que se dedicam a essa atividade. Na maior parte das vezes, os profissionais da saúde voltam mais sua atenção para os procedimentos a serem realizados em benefício do "ser doente", não contemplando as necessidades e particularidades desses familiares que são extremamente importantes para o sucesso das abordagens das equipes de saúde.

Ao conduzir um estudo qualitativo de caráter fenomenológico, com o intuito de compreender o que é cuidar de um idoso dependente por meio do olhar de seus familiares, foram evidenciadas quatro unidades de significado que permitiram observar vários aspectos dessa vivência: o contato inicial com o cuidar e as formas de enfrentamento; as dificuldades associadas ao cuidado; os sentimentos envolvidos no ato de cuidar; e as sobrecargas do cuidador familiar. 
O objetivo deste estudo é apresentar os resultados relacionados à sobrecarga física e mental relatada pelos cuidadores familiares de idosos dependentes, de modo a fornecer informações que possam contribuir de forma positiva para a complementação do trabalho das equipes de saúde voltado para os idosos dependentes, levando em consideração que ele também deve se voltar para a assistência dos cuidadores familiares.

\section{METODOLOGIA}

Trata-se de estudo descritivo-exploratório, qualitativo, de caráter fenomenológico. Foram realizadas entrevistas, no período de junho a julho de 2010, com os cuidadores familiares dos idosos dependentes assistidos pela Pastoral da Pessoa Idosa do Bairro Neva, do município de Cascavel-PR. Foi considerado dependente o indivíduo que necessitava de ajuda parcial ou total para a realização de suas atividades de vida diária. ${ }^{9}$ Foram entrevistados todos os familiares envolvidos nas atividades de cuidado do idoso dependente - ou seja, nas famílias onde havia mais de um cuidador familiar, foi entrevistada mais de uma pessoa.

Durante o período da coleta de dados, a Pastoral estava acompanhando 430 idosos, sendo que dentre estes havia 15 (3,49\%) indivíduos dependentes. Foram contatadas 13 famílias, pois havia dois idosos dependentes em duas famílias. Em somente dois domicílios, o cuidador principal dividia suas tarefas relacionadas ao cuidado dos idosos dependentes com outros familiares. Sendo assim, foram entrevistados 15 cuidadores com idade média de 57 anos, com um mínimo de 32 e um máximo de 81 anos.

Dentre os entrevistados, 13 (86,67\%) eram do sexo feminino e apenas dois $(13,33 \%)$ do sexo masculino. Em relação ao grau de parentesco, em dez casos eram os filhos que prestavam os cuidados ao idoso; em quatro casos, os cônjuges; e em apenas um caso os cuidados eram desempenhados pelo irmão do idoso assistido.
A coleta de dados foi realizada por meio de entrevista semiestruturada, baseada na seguinte pergunta orientadora: "Para você, o que é cuidar de um(a) idoso(a) dependente?". As entrevistas foram gravadas e transcritas na íntegra para serem analisadas. Todos que concordaram em participar leram e assinaram o termo de consentimento livre e esclarecido (TCLE).

As entrevistas foram agendadas previamente e realizadas nas residências dessas pessoas, por se considerar que neste local elas se sentiriam mais à vontade para falar sobre suas vivências. Para melhor caracterização desses cuidadores e da dependência dos idosos, foi aplicado também um questionário. Após a visita a cada residência, foram anotadas em um "diário de campo" todas as informações e impressões obtidas, com a finalidade de complementar os dados das entrevistas.

As entrevistas transcritas foram identificadas pela letra "C" (cuidador) e por números, e sua análise se baseou em alguns direcionamentos da fenomenologia: ${ }^{10}$ leitura de cada texto transcrito na íntegra; leitura de cada texto, atendo-se à questão orientadora, visando a identificar "afirmações" que apresentassem significados relevantes; agrupamento das "afirmações" com temáticas semelhantes, discriminando assim as "unidades de significado"; e por fim, para conhecer a essência do fenômeno estudado, foi realizada a interpretação (análise compreensiva) dessas "unidades de significado". Para a discussão dos dados, foi utilizado o referencial teórico relacionado ao estudo.

Esta pesquisa foi aprovada pelo Comitê de Ética em Pesquisas (CEP) da Universidade Estadual do Oeste do Paraná (UNIOESTE), sob o parecer n. 101/2010.

\section{RESULTADOS}

A maioria dos cuidadores entrevistados havia completado o Ensino Médio (46,67\%), exercia algum tipo de atividade remunerada (46,67\%) e tinha renda familiar de dois a cinco salários 
mínimos (46,67\%). O tempo que eles se dedicavam aos cuidados variou de três meses a 22 anos.

Em relação à população idosa assistida por esses cuidadores, dez eram do sexo feminino $(66,67 \%)$ e cinco do sexo masculino (33,33\%). Apresentavam em média 74 anos, com um mínimo de 62 e o máximo de 95 anos. A maioria era total ou parcialmente dependente de outras pessoas para atividades relacionadas à higiene pessoal (80\%), para se alimentarem (53,33\%), vestirem-se (86,67\%), locomoverem-se e/ou deambularem $(93,33 \%)$.

Por meio da leitura exaustiva das falas e sua análise, foi possível observar que o cuidador familiar de idosos dependentes é uma pessoa sobrecarregada em vários aspectos. Isso acontece pelo acúmulo de funções e os tipos de tarefas que ele assume. Além das responsabilidades relacionadas ao ato de cuidar em si, existe ainda as responsabilidades relacionadas à casa e à família.

É muita coisa sabe, é muita coisa. É serviço, é filho, é marido. E a gente se estressa, tem hora que estressa sabe. (C3)

Então, para ir ao mercado eu tenho que ir, pra pagar uma luz e água eu tenho que ir, pra ir pagar uma prestação nas lojas eu tenho que ir, tudo eu tenho que resolver, todos os problemas en tenho que resolver. Então pra mim, fica difícil, dá muito trabalho. (C2)

Diante das entrevistas, percebe-se que o idoso dependente necessita muitas vezes de atenção em tempo integral. Isso ocorre pela dificuldade que ele tem em realizar determinadas atividades de vida diária, como os cuidados com a sua higiene, alimentação, deambulação e administração de medicamentos. Dessa forma, o cuidador sentese "obrigado" a estar disponível o dia todo para auxiliá-lo. E mesmo nos casos em que o idoso não depende totalmente do auxílio de outras pessoas, o familiar precisa estimular verbalmente a realização das tarefas do seu cotidiano.

A gente tem que ficar sempre ali, sempre. (C2)

Você tem que ficar ali 24 horas bem dizer por dia, é de manhã, de meio-dia, de tarde, remédio, é tudo na hora certa. (C1)
Porque é tudo, é aquele tal negócio, uma idade como eles estão, eles não fazem as coisas direito. Então, você tem que estar empurrando as coisas sabe. (C14)

Nota-se também que a execução contínua de atividades relacionadas ao cuidado que exige força, como transferências e banhos, resulta em cansaço físico e desgaste psicológico para o cuidador, que na maior parte dos casos não possui ajuda de outro familiar para realizá-las.

É, a gente ter que pegar, levar no banheiro, porque ele não vai sozinho. Se está deitado, tem que levantar [...7. (C13)

Para mim trabalhar, assim, pegar ela, a gente acha difícil porque ela é pesada, nós somos pequenininhas. Então acho assim, é muito difícil. (C15)

Mas não é fácil também você pegar cadeiras de rodas, colocar dentro do carro, tirar cadeira de rodas, colocar ele sentado [...]. Eles colocam muita responsabilidade em cima de mim, estão me sufocando, me sufoca. (C3)

Quando a pessoa que cuida também possui restrições na sua saúde, a sobrecarga é maior ainda. Alguns cuidadores entrevistados eram também idosos que apresentavam alterações do seu estado de saúde. Mesmo assim, continuavam a cuidar dos seus familiares por saberem que as limitações apresentadas por eles eram maiores que as suas.

\section{Eu também não tenho saúde. Passar a noite sem dormir assim, é muito difícil pra mim, tenho problema no coração, problema de pressão. Mas fazer o que. Tem que olhar. Vou tomando meu remédio, e olhando ele. (C4). \\ A gente, não tem muita saúde também. Eu sofro muito da coluna, principalmente do estômago, tenho problema de pressão. Não tenho muita saúde também. (C10).}

Tudo na vida do cuidador é planejado e executado em função das atividades relacionadas ao cuidado do idoso. Muitos começam a deixar sua própria vida de lado e acabam por esperar o momento em que essa responsabilidade terminará, para voltar a viver normalmente.

Seu tempo fica assim, tudo ali, entendeu? (C6)

Mas, enquanto ela estiver viva, eu só peço sempre pra Deus me dar saúde para depois um dia, eu poder ainda viver um pouco a vida. (C1). 
Observa-se nas falas que a dependência do idoso mantém o cuidador a maior parte do tempo no domicílio, pois, por medo e preocupação, prefere não deixar seu familiar sozinho, o que resulta no isolamento social de ambos. Em outros casos, a dificuldade de deambulação/ locomoção e as barreiras arquitetônicas que dificultam a acessibilidade de locais relacionados ao lazer acentuam esse isolamento.

E a gente fica preso. Porque igual ela, ela ficou presa e eu também. Me prendeu também, porque en não posso, bem dizer não tenho liberdade para passear, tipo passear na casa de um parente longe aí, ir ficar quatro, cinco dias, pra mim é difícil. (C2)

E sair de dentro de casa com ele assim, não é fácil. (C13)

Ele para levar, não consegue subir, ir nos lugares [...]. É muito complicado, é difícil! (C3)

Em muitos casos, observou-se que a família transfere a responsabilidade do cuidar para uma única pessoa, que não conta com a ajuda de outras para efetuar suas tarefas. Por causa disso e pelo fato de o cuidador ter que mudar sua vida em função do idoso, de ter a necessidade de conciliar a sua vida em família com o cuidar do idoso dependente, de ter que planejar suas atividades dependendo da necessidade do outro, de não saber lidar com certas situações de convivência e de nunca ter tempo para descanso e lazer, a sobrecarga emocional é uma constante em suas vidas. Em alguns casos, os entrevistados precisavam de tratamento medicamentoso específico para ansiedade e depressão.

Eu não sei, tem horas que, eu já falei para os filhos deles, tem hora que eu tenho vontade de mudar daqui também, igual eles. Mudar e só vir visitar uma vez. por mês, quinze minutos só, não passar mais de meia hora ali não. Daí é bom. Daí você não se preocupa se ficou doente durante a semana, se precisou levar para o médico, o que que precisou, não tem responsabilidade, tem hora que eu penso isso. (C3).

Eu acho o pior de tudo, isso, que mexe muito com o meu emocional sabe, aí você vai limpar, por exemplo, ela fez cocô, você não vai dar banho toda a hora que ela fez cocô, principalmente no inverno, aí você vai limpar, ela não gosta que você limpe, a água tem que estar bem na temperatura normal, senão ela já reclama. (C15)
E é sempre tentando controlar, controlar e largar dos remédios, e controlando, mas no começo foi muito difícil. (C15)

É complicado eu acho assim. Você tem que encaixar um monte de coisa, tem que encaixar teus filhos, que tem uma idade diferente, na adolescência, eles não entendem muita coisa, e eles também não podem abrir mão da vida toda deles, porque o tempo vai passando. Passa para a gente, passa para eles. (C6)

\section{DISCUSSÃO}

Em geral, os cuidadores familiares são do sexo feminino, como pode ser observado neste estudo. Isso pode ser explicado pelo fato de que, culturalmente, a sociedade atribui à figura da mulher a responsabilidade das tarefas da casa, da educação e cuidado dos filhos e da saúde dos membros de sua família. ${ }^{11,12}$

A mulher, ao cuidar de uma pessoa dependente, passa a agregar novas tarefas ao seu cotidiano. Grande parte das cuidadoras reside com o idoso e, por isso, somam a suas atividades de cuidar, as atividades domésticas de mãe, esposa e avó, gerando acúmulo de trabalho em casa e sobrecarga nos diversos domínios da sua vida. ${ }^{13-15}$

Quanto à relação de parentesco entre o cuidador e o idoso, geralmente são os cônjuges que assumem essas atividades, por isso, na maior parte das vezes é possível encontrar uma pessoa que está envelhecendo cuidando de outra que está passando pela mesma experiência, assim como foi evidenciado nas famílias visitadas. ${ }^{16}$ Os cuidadores mais velhos são mais susceptíveis à sobrecarga e a problemas de saúde, visto que as alterações decorrentes do processo de envelhecimento estão cada vez mais presentes e se somam ao desgaste físico e psicológico que o dia a dia de deveres e responsabilidades acarreta. ${ }^{17}$

Desta forma, à medida que o tempo vai passando, o cuidador tem cada vez mais dificuldade para cuidar de si mesmo e do outro. ${ }^{18}$ Tem que aprender a lidar com o seu próprio envelhecimento e também com o envelhecimento, a dependência e a proximidade da morte do familiar idoso dependente. ${ }^{12}$ 
O nível de dependência física dos idosos está diretamente associado à sobrecarga do cuidador. ${ }^{19}$ Quanto maior o número de atividades que os cuidadores desenvolvem, maior o esforço $\mathrm{e}$, consequentemente, maior o desgaste físico e mental. ${ }^{20}$ Esse desgaste, aliado às atividades e preocupações diárias do cotidiano, as próprias limitações e incapacidades do cuidador, tornam a vida em si mais penosa, podendo trazer danos e maiores riscos a sua saúde. Pode-se então dizer que existe correlação entre a qualidade de vida do cuidador e a situação funcional do indivíduo que ele assiste. ${ }^{18}$

A maior parte dos entrevistados deste estudo cuidava de indivíduos que eram parcial ou totalmente dependentes para executar várias atividades básicas de vida diária, o que pode ter contribuído para os relatos de intensa sobrecarga física e mental.

A presença da incapacidade funcional pode fazer com que o familiar esteja envolvido de 19 a 24 horas por dia com o cuidado do idoso. Atividades como alimentação, higiene, locomoção/deambulação podem consumir mais de cinco horas por dia para serem realizadas com o auxílio do cuidador. ${ }^{13,21,22} \mathrm{~A}$ execução de tarefas de forma contínua e repetitiva; a falta de informações e preparo para cuidar do idoso; e a falta de uma estrutura física e equipamentos adequados para auxiliar a realização de tarefas mais pesadas ${ }^{23,24}$ podem agravar ainda mais o cansaço físico e psicológico do cuidador.

O cuidado de idosos dependentes exige dedicação quase que exclusiva, fazendo com que o familiar deixe de vivenciar outras experiências interpessoais e sociais, o que acaba afetando seu modo de encarar a própria vida. ${ }^{25}$ A alteração das suas atividades sociais, as mudanças nos relacionamentos familiares e no círculo de amizades são fatores limitantes para a sua vida social. ${ }^{18}$ Como consequências, surgem o isolamento social ${ }^{14}$ e alterações no seu estado do humor. ${ }^{13,20,26}$ A sobrecarga de sentimentos conflituosos é uma constante, sendo que muitas vezes a depressão pode estar presente na vida dessas pessoas. ${ }^{23}$
O cuidador pode apresentar alto nível de ansiedade e depressão, tanto pelo desempenho de novos papéis e tarefas associadas ao problema do idoso, quanto por constatar que sua família está sendo afetada por tudo isso. Ele é continuamente "testado" em sua capacidade de escolha e adaptação à nova realidade, que exige dedicação, responsabilidade, paciência e renúncia. ${ }^{24,27,28}$

Pelos fatores já descritos, a experiência de cuidar de idosos tem sido caracterizada como uma situação estressante que afeta a saúde física e psicológica do cuidador. ${ }^{29} \mathrm{O}$ estresse crônico pode promover alterações imunológicas no familiar cuidador que contribuem para o aumento da sua vulnerabilidade a doenças, ${ }^{30}$ além de poder acarretar alterações de suas habilidades cognitivas, que por sua vez comprometem o cuidado prestado ao idoso, bem como o seu autocuidado. ${ }^{31,32}$ Por causa dessas consequências, a família pode se sobrecarregar financeiramente, devido os gastos com a saúde do idoso e também do cuidador.

Pelo processo de envelhecimento populacional mundial, o número de idosos vem aumentando. Sendo assim, os profissionais da saúde necessitam estar preparados para atender a essa demanda. Não basta somente o conhecimento técnico-científico sobre como realizar a prevenção, tratamento e reabilitação dos agravos de saúde que acometem essa faixa etária específica. As estratégias em saúde das equipes devem contemplar também as pessoas que mais se envolvem com a continuidade do tratamento desses idosos no seu domicílio, que são seus familiares. ${ }^{16,33}$

A saúde de um indivíduo - no caso deste estudo, dos idosos - depende do equilíbrio entre fatores biológicos, psicológicos e sociais. Quando algum desses fatores está comprometido, afeta os demais, facilitando o surgimento do desequilíbrio. É nesse momento que o profissional da saúde deve intervir, visando a favorecer e promover condições para que esse indivíduo e os familiares envolvidos com os seus cuidados e sua saúde tenham condições de transformar aspectos negativos em positivos, melhorando a qualidade de vida de todos. ${ }^{1}$ 
Os comprometimentos da qualidade de vida física e mental do cuidador interferem de forma negativa no processo de reabilitação da pessoa assistida. Para que o cuidador não abandone seu papel e se mantenha saudável e com qualidade de vida, é de fundamental importância que ele aprenda a se adaptar a essa nova condição. ${ }^{11}$ Nessa perspectiva, é importante reconhecer que abordar a família de um paciente é fundamental no processo de prestação de serviços em saúde. Ela contribui para a construção de um ambiente favorável para a recuperação da saúde do idoso, evitando, na medida do possível, hospitalizações, asilamentos ou outras institucionalizações. ${ }^{21}$

Para tentar diminuir a sobrecarga e auxiliar esses familiares, é necessário que as equipes de saúde promovam orientações e esclarecimentos quanto ao que os idosos apresentam e as melhores maneiras para se exercer as atividades relacionadas ao cuidado, no domicílio. Além disso, os profissionais devem levar em consideração que o familiar acaba se envolvendo muito com o problema do idoso que assiste, precisando compartilhar suas dúvidas e anseios. É preciso estar disponível para ouvir esses cuidadores, pois eles consideram a equipe multidisciplinar uma fonte de apoio importante. ${ }^{16}$

Há a necessidade de programas de apoio aos cuidadores e às famílias, com o intuito de reconhecer se existem ou não entre os cuidadores, distúrbios emocionais que necessitam de atendimento especializado. Esses programas também apresentam outros objetivos voltados para o cuidador, como reduzir o seu isolamento e melhorar seu desempenho; mobilizar recursos e suportes físicos e sociais para ajudá-lo; aumentar sua capacidade de resolução de problemas e o desenvolvimento de habilidades; aumentar seu conhecimento sobre o processo de envelhecimento e os problemas de saúde específicos da faixa etária a quem ele dedica os cuidados. ${ }^{6,33}$

Nesse contexto, também merece destaque o incentivo do estabelecimento e/ou fortalecimento do apoio por meio do suporte social, que está associado às relações interpessoais, grupais ou comunitárias que concedem ao indivíduo um sentimento de proteção e auxílio, capaz de propiciar bem-estar psicológico, redução do estresse, ${ }^{14}$ depressão e ansiedade. ${ }^{6}$

Cuidadores que se engajam em atividades na comunidade e participam de grupos de apoio compostos por famílias em situações semelhantes, adaptam-se melhor à função de cuidador e apresentam níveis mais baixos de depressão e maiores de satisfação. Acredita-se que, em grupo, o familiar cuidador seja capaz de se reconhecer no outro, fazer questionamentos a si próprio e buscar respondê-los a partir dessas experiências. Há também o efeito terapêutico resultante da identificação entre pessoas que compartilham um mesmo problema, auxiliando esses cuidadores no enfrentamento da situação vivenciada. ${ }^{26,34}$

A equipe de saúde deve avaliar a dinâmica familiar com atitude de respeito e valorização frente a suas particularidades, buscando reconhecer e acompanhar o membro responsável pelo cuidado e pondo em prática o conceito de que saúde não é um estado, mas um processo multidimensional, que envolve sistemas biológicos, sociais, culturais e ambiental. ${ }^{26}$

É imprescindível que o cuidador receba apoio de outras pessoas da família, pois isso contribui para a diminuição da sobrecarga. Dessa forma, a adaptação à rotina do cuidador é facilitada, e suas atividades pessoais não precisam ser colocadas de lado. ${ }^{35}$ Cabe também ao profissional da saúde fornecer o estímulo para a identificação de outros cuidadores na família. ${ }^{23}$ Recomenda-se que todos os membros familiares sejam incentivados a atuarem em conjunto com o cuidador principal do idoso dependente, dividindo tarefas e procurando proporcionar um clima de satisfação no ambiente familiar. ${ }^{24}$

\section{CONCLUSÃO}

A análise compreensiva das falas dos indivíduos que participaram da pesquisa evidenciou que muitos deles são sobrecarregados física e mentalmente. Foram identificadas algumas características dos cuidadores que favorecem o aparecimento da sobrecarga, como o 
fato de ele pertencer ao gênero feminino, possuir idade avançada e se responsabilizar sozinho por todos os cuidados. Em relação às características do idoso que podem estar contribuindo para esta sobrecarga, pode-se citar o nível de sua capacidade funcional.

Ao prestar atendimento aos idosos, muitas vezes o profissional está preocupado somente com a enfermidade, com a técnica a ser executada e com o corpo biológico, não levando em consideração a importância de outros fatores que podem influenciar o processo saúde-doença. $\mathrm{O}$ profissional de saúde deve, além do conhecimento específico sobre geriatria e gerontologia, conhecer todos os aspectos que envolvem a vida do "ser" que está tratando, para que o atendimento se torne mais completo e, consequentemente, mais humanizado, contribuindo assim para o sucesso da intervenção.

\section{REFERÊNCIAS}

1. Duarte VB, Santana MG, Soares MC, Dias DG, Thofern MB. A perspectiva do envelhecer para o ser idoso e sua família. Família, Saúde e Desenvolvimento 2005; 7(1): 42-50.

2. Neri AL, Fortes ACG. A dinâmica do estresse e enfrentamento da velhice e sua expressão no prestar cuidados a idosos no contexto da família. In: Freitas EV, et al. Tratado de Geriatria e Gerontologia. Rio de janeiro: Guanabara Koogan; 2006.

3. Nunes LM, Portella MR. O idoso fragilizado no domicílio: a problemática encontrada na atenção básica em saúde. Boletim da Saúde 2003; 17(2): 109-21.

4. Figueiredo MLF, et al. Diagnósticos de enfermagem do idoso acamado no domicílio. Rev Bras Enferm. 2008; 61(4): 464-9.

5. Rosa TEC, Benício MHD, Latorre MRDO, Ramos LR. Fatores determinantes da capacidade funcional entre idosos. Rev Saude Publica. 2003; 37(1): 40-8.

6. Diogo MJD'E, Ceolim MF, Cintra FA. Orientações para idosas que cuidam de idosos no domicílio. Rev Bras Enferm. 2005; 39(1): 97-102.

7. Cattani RB, Girardon-Perlini NMO. Cuidar do idoso doente no domicílio na voz de cuidadores familiares. Revista eletrônica de enfermagem 2004; 6(2): 254-71.
Sendo assim, as equipes de saúde que lidam com pessoas idosas devem voltar seu olhar não somente para a prestação de serviços especializados a essas pessoas, mas também para a assistência dos familiares responsáveis pelos cuidados. Esses familiares merecem atenção especial nesse contexto, por vivenciarem uma situação de estresse crônico que acaba afetando sua saúde física e psicológica, e por serem os responsáveis pela continuidade do tratamento prestado aos idosos, quando estes se encontram em seus domicílios.

Para complementar os dados do presente estudo, sugere-se para trabalhos futuros a abordagem do idoso dependente, para melhor compreender a relação entre o cuidador e a pessoa que está sendo cuidada. Sugere-se, ainda, a realização de estudos que avaliem de forma mais detalhada a influência do estresse crônico na saúde desses cuidadores.

8. Karsch UM. Idosos dependentes: famílias e cuidadores. Cad Saude Publica 2003; 19(3): 861-6.

9. Caldas CP. Envelhecimento com dependência: responsabilidades e demandas da família. Cad Saude Publica 2003; 19(3): 773-81.

10. Giorgi A. Phenomenology and Psychological Research. Pittsburgh: Duquesne University Press; 2003.

11. Panhoca I, Pupo ACS. Cuidando de quem cuida: avaliando a qualidade de vida de cuidadores de afásicos. Revista CEFAC : actualização científica em fonoaudiologia. 2010; 12(2): 299-307.

12. Salgueiro H, Lopes M. A dinâmica da família que coabita e cuida de um idoso dependente. Rev Gaucha Enferm. 2010; 31(1): 26-32.

13. Gonçalves LHT, Alvarez AM, Sena ELS, Santana LWS, Vicente FR. Perfil da família cuidadora de idoso doente/fragilizado do contexto sociocultural de Florianópolis, SC. Texto \& contexto enfermagem / UFSC. 2006; 15(4): 570-7.

14. Mendonça FF, Garanhani ML, Martins VL. Cuidador familiar de sequelados de acidente vascular cerebral: significado e implicações. Physis 2008; 18(1): 143-58. 
15. Machado ALG, Jorge MSB, Freitas CHA. A vivência do cuidador familiar de vítima de Acidente Vascular Encefálico: uma abordagem interacionista. Rev Bras Enferm. 2009; 62(2): 246-51.

16. Moreira MD, Caldas CP. A importância do cuidador no contexto da saúde do idoso. Escola Anna Nery revista de enfermagem. 2007; 11(3): 520-5.

17. Amendola F, Oliveira MAC, Alvarenga MRM. Qualidade de vida dos cuidadores de pacientes dependentes no programa de saúde da família. Texto \& contexto enfermagem / UFSC. 2008; 17(2): 266-72.

18. Rodrigues SLA, Watanabe HAW, Derntl AM. A saúde de idosos que cuidam de idosos. Rev Esc Enferm USP 2006; 40(4): 493-500.

19. Bocchi SCM. Vivenciando a sobrecarga ao vir-aser um cuidador familiar de pessoa com acidente vascular cerebral (AVC): análise do conhecimento. Rev Lat Am Enfermagem 2004; 12(1): 115-21.

20. Lemos ND, Gazzola JM, Ramos LR. Cuidando do paciente com Alzheimer: o impacto da doença no cuidador. Saúde e sociedade. 2006; 15(3): 170-9.

21. Nascimento LC; Moraes ER; Silva JC; Veloso LC; Vale ARMC. Cuidador de idosos: conhecimento disponível na base de dados LILACS. Rev Bras Enferm. 2008; 61(4): 514-7.

22. Pinto MF, Barbosa DA, Ferreti CEL, Souza LF, Fram DS, Belasco AGS. Qualidade de vida de cuidadores de idosos com doença de Alzheimer. Acta paulista de enfermagem.. 2009; 22(5): 652-7.

23. Luzardo AR, Waldman BF. Atenção ao familiar cuidador do idoso com doença de Alzheimer. Acta scientiarum. Health sciences. 2004 Jan-Jun; 26 (1): b135-45.

24. Rocha MPF, Vieira MA, Sena RR. Desvelando o cotidiano dos cuidadores informais de idosos. Rev Bras Enferm. 2008; 61(1): 801-8.

25. Freire GD, Campos DR, Boemer MR. Compreendendo o paciente gravemente enfermo e sua família na realidade domiciliar. Rev Gaucha Enferm. 2004; 25(6): 346-56.
26. Machado ALG, Freitas CHA, Jorge MSB. O fazer do cuidador familiar: significados e crenças. Rev Bras Enferm. 2007; 60(5): 530-4.

27. Luzardo AR, Gorini MIPC, Silva APSS. Características de idosos com doença de Alzheimer e seus cuidadores: uma série de casos em um serviço de neurogeriatria. Texto \& contexto enfermagem / UFSC. 2006; 15(4): 587-94.

28. Resende MCF, Dias EC. Cuidadores de idosos: um novo / velho trabalho. Physis. 2008; 18(4): 785-800.

29. Pinquart M, Sörensen S. Differences between caregivers and noncaregivers in psychological health and physical health: a meta-analysis. Psychol Aging. 2003; 18(2): 250-67.

30. Bauer ME, Vedhara K, Perks P, Wilcock GK, Lightman SL, Shanks N. Chronic stress in caregivers of dementia patients is associated with reduced lymphocyte sensitivity to glucocorticoids. J Neuroimmunol. 2000 Feb 1;103(1):84-92.

31. Balardin JB, Palma KAXA, Garcia Junior ME, Bromberg E. Déficits cognitivos em cuidadores de pacientes com demência. RBCEH Revista Brasileira de Ciências do Envelhecimento Humano. 2007; 4(2): 55-64.

32. Palma KA, Balardin JB, Vedana G, de Lima Argimon II, Luz C, Schröder N. Emotional memory déficit and its psychophysiological correlate in family caregivers of patients with dementia. Alzheimer Dis Assoc Disord. 2011; 25(3): 262-8.

33. Cerqueira ATAR, Oliveira NIL. Programa de apoio a cuidadores: uma ação terapêutica e preventiva na atenção à saúde dos idosos. Psicologia--USP. 2002; 13(1): 133-50.

34. Cruz MN, Hamdan AC. O impacto da doença de Alzheimer no cuidador. Psicol Estud. 2008; 13(2): 223-9.

35. Lopes MCL, Marcon SS. A hipertensão arterial e a família: a necessidade do cuidado familiar. Rev Esc Enferm USP. 2009; 43(2): 343-50. 